Rapid Reviews COVID-19

\title{
Review 2: "LOW BIRTH WEIGHT AS A RISK FACTOR FOR SEVERE COVID-19 IN ADULTS"
}

Anders Hviid ${ }^{1}$

${ }^{1}$ Professor, Statens Serum Institut, Department of Epidemiology Research, Denmark

Published on: Dec 03, 2020

DOI: $10.1162 / 2 \mathrm{e} 3983 f 5 . a 6 d 57 b 5 d$

License: Creative Commons Attribution 4.0 International License (CC-BY 4.0). 


\section{RR:C19 Evidence Scale rating by reviewer:}

- Potentially informative. The main claims made are not strongly justified by the methods and data, but may yield some insight. The results and conclusions of the study may resemble those from the hypothetical ideal study, but there is substantial room for doubt. Decision-makers should consider this evidence only with a thorough understanding of its weaknesses, alongside other evidence and theory. Decisionmakers should not consider this actionable, unless the weaknesses are clearly understood and there is other theory and evidence to further support it.

$* * * * * * * * * * * * * * * * * * * * * * * * * * * * * * * * * * * * * * *$

\section{Review:}

This study appears to have two goals: I) to test the hypothesis that LBW is a risk factor for severe COVID-19 and II) to evaluate whether LBW is an important prognostic factor for severe COVID-19.

With respect to I) the authors estimate univariate ORs of ICU admission for a number of covariates (age, sex, BMI, comorbidity, LBW, etc.) in their dataset. They then conduct a multivariate analysis on the variables, which reached statistical significance. This is a problematic approach, which increases the type I error rate. The authors then proceed to evaluate the prognostic performance of their model and conclude that LBW is an important prognostic factor for severe COVID-19 from an AUROC of 0.74 in a validation dataset. I see no analysis supporting this conclusion. LBW can be a risk factor without being an important prognostic factor. A multivariate model without LBW might well produce a comparable AUROC.

The real-world usability for LBW as a prognostic marker is difficult to see. I would imagine that this type of information would not be available in most scenarios and thus would not be much help in risk stratification.

It is interesting that LBW could be a risk factor for severe COVID-19, but I would not conclude anything other than further research is needed. 